\title{
Gastos público e privado com saúde no Brasil e países selecionados
}

\author{
Public and private health expenditures in Brazil and selected \\ countries
}

Juliana Oliveira Figueiredo', Nilia Maria de Brito Lima Prado², Maria Guadalupe Medina ${ }^{\mathbf{3}}$, Jairnilson Silva Paim 4

DOI: 10.1590/0103-11042018S203

1 Universidade Federal da Bahia (UFBA), Instituto de Saúde Coletiva (ISC), Programa de Pós-

Graduação em Saúde Coletiva (PPGSC) -

Salvador (BA), Brasil. Orcid: https://orcid. org/0000-0002-61628251

julicajaiba@hotmail.com

2 Universidade Federal da Bahia (UFBA), Instituto Multidisciplinar em Saúde (IMS), Programa de

Pós-Graduação em Saúde Coletiva (PPGSC) - Vitória da Conquista (BA), Brasil. Orcid: https://orcid. org/0000-0001-8243-

5662

nilia.ufba@gmail.com

3 Universidade Federal da Bahia (UFBA), Instituto de Saúde Coletiva (ISC),

Programa de PósGraduação em Saúde Coletiva (PPGSC) Salvador (BA), Brasil. Orcid: https://orcid. org/0000-0001-72832947

medina@ufba.br

4 Universidade Federal da Bahia (UFBA), Instituto de Saúde Coletiva (ISC), Programa de PósGraduação em Saúde Coletiva (PPGSC) -

Salvador (BA), Brasil.

Orcid: https://orcid.

org/0000-0003-0783-

262X

jairnil@ufba.br
RESUMO O estudo buscou analisar os indicadores dos gastos público e privado no Brasil e países selecionados no período de 2000 a 2014. Foram acessadas bases de domínio público de diferentes fontes via internet. Os indicadores referentes aos gastos públicos em saúde decresceram ou não apresentaram aumento e os referentes aos gastos privados mantiveram-se estáveis. O Brasil foi o país com menor participação do gasto público em saúde, diferentemente do que ocorreu em países com sistemas de saúde similares, universais e públicos. Os dados demonstraram que o gasto com a saúde privada é relevante no orçamento familiar. Tais achados apontam para uma redução do papel do Estado como provedor e financiador das ações e serviços públicos de saúde. Como resultado das medidas de austeridade, percebeu-se queda nos gastos com saúde, por exemplo, na porcentagem do Produto Interno Bruto dedicado à saúde, estabilizada ou diminuída ligeiramente em países da Europa. No Brasil, com a implantação do 'novo regime fiscal', espera-se que os gastos públicos com saúde apresentem maior redução, dada a severidade da política de austeridade.

PALAVRAS-CHAVE Sistema Único de Saúde. Financiamento da assistência à saúde. Gastos em saúde.

ABSTRACT The study sought to analyze the indicators of public and private spending in Brazil and selected countries from 2000 to 2014. Public domain databases from different sources obtained through the Internet were accessed. The indicators related to public health expenditures decreased or did not increase and those related to private spending remained stable. Brazil was the country with the lowest share of public health spending, differently from what occurred in countries with similar, universal and public health systems. The data showed that spending on private health is relevant in the family budget. These findings point to a reduction of the role of the State as provider and financier of public health actions and services. As a result of the austerity measures, a decline in health spending was perceived, for example, in the percentage of Gross Domestic Product devoted to health, stabilized or slightly decreased in European countries. In Brazil, with the implementation of the 'new fiscal regime', public health expenditures are expected to decline more, given the severity of the austerity policy.

KEYWORDS Unified Health System. Healthcare financing. Health expenditures. 


\section{Introdução}

Com a Constituição da República de 1988, a saúde passou a ser reconhecida como um direito social, cabendo ao poder público a obrigação de garanti-lo'. Esta conquista política e social pode ser atribuída a diversas lutas e esforços empreendidos pelo Movimento da Reforma Sanitária². Assim, a Constituição estabeleceu as bases para a criação do Sistema Único de Saúde (SUS), posteriormente regulamentado por meio das Leis $\mathrm{n}^{\circ} 8.080 / 90$ e $\mathrm{n}^{\circ} 8.142 / 90$, assim como pela Lei Complementar $n^{0} 141 / 12^{3-5}$.

No que se refere ao financiamento, a Lei $n^{\circ}$ 8.142/90 dispõe sobre as transferências intergovernamentais de recursos financeiros na área da saúde ${ }^{4}$, enquanto a Lei Complementar $n^{\circ}$ 141/12 regulamenta a Emenda Constitucional $n^{\circ} 29$, fixando a participação da União, Estados e Municípios no orçamento do setor ${ }^{5}$. O sistema de saúde brasileiro é financiado por meio de impostos gerais, contribuições sociais, desembolso direto e gastos dos empregadores com saúde. Ou seja, o Brasil possui um sistema de saúde inteiramente financiado por receitas fiscais gerais. O financiamento do SUS tem como fontes os recursos dos impostos das três esferas de governo, compreendidas as transferências constitucionais para os Estados e Municípios ${ }^{2,6}$.

O Brasil, apesar de ter por mandato constitucional um sistema público de saúde de acesso universal, apresenta o gasto privado em saúde superior ao gasto público. Países com sistemas de saúde similares, ou seja, universais e públicos, gastam, em média, $8 \%$ do Produto Interno Bruto (PIB). No Brasil, em 2014, foram gastos com o SUS apenas $3,9 \%$, considerando a participação da União, Estados e Municípios?.

O financiamento do SUS não tem sido suficiente para assegurar recursos financeiros adequados para o sistema público, destinando, portanto, menor volume para atendimento às necessidades de saúde da população do que aquele previsto quando o sistema foi criado ${ }^{2}$.
Para agravar tal situação, no Brasil, foram adotadas as políticas de austeridade fiscal. Dentre as ações mais recentes adotadas para a limitação de despesas, destaca-se a Emenda Constitucional $\mathrm{n}^{\circ}$ 95, de 2016 (EC 95/16), criando o Regime Fiscal, que estipulou um teto para a despesa primária da União e o congelamento do gasto com saúde em valores reais de 2016, por 20 anos. Tal medida reduziria o valor do PIB, do governo federal, destinado à saúde, à medida que a economia crescesse, bem como diminuiria o gasto per capita, desconsiderando que a população de idosos no Brasil dobrará nesse mesmo período 8 .

O País vivencia um período de crise econômica e o foco das discussões, mais uma vez, se concentra no corte e/ou contenção de gastos públicos, especialmente dos gastos sociais, vistos por alguns como descontrolados e causadores do deficit público ${ }^{8}$. Na prática, o ajuste fiscal implementado no Brasil pode gerar a redução da participação do Estado na oferta de bens e serviços à população, com a redução dos gastos públicos, o que poderá conduzir a um cenário de redução na participação das despesas primárias no PIB de cerca de $20 \%$, em 2016, para $16 \%$ a $12 \%$ do PIB, até $2026^{6}$.

No contexto mundial, a crise econômica de 2008 implicou um conjunto de políticas de austeridade implantadas, principalmente, em países da Europa, envolvendo a limitação de direitos sociais e reformas no sistema de saúde. Entre esses países, é possível citar Grécia, Portugal e Espanha, que enfrentaram crises graves a partir do referido ano ${ }^{9-11}$. No setor saúde, essas reformas envolviam cortes orçamentários com restrições de serviços, aumento de copagamento, transferência de custos para os usuários, diminuição de responsabilidades por parte do Estado e nas formas de privatização ${ }^{\mathbf{1 2}}$.

Em países de alta renda, membros da Organização para a Cooperação e o Desenvolvimento Econômico (OCDE), a crise econômica também teve efeitos 
negativos sobre o sistema público de saúde e as condições de saúde da população $0^{10,13,14}$.

Desse modo, os indicadores referentes aos gastos em saúde precisam ser acompanhados para fundamentar políticas e possibilitar o debate com evidências científicas. Faz sentido, também, examinar o sistema de saúde brasileiro para além do SUS, tendo em vista a expansão do setor privado ${ }^{15}$, devido a incentivos fiscais por parte do governo federal. Sem exigir a contrapartida, concordando com Melo ${ }^{16}$, tal prática tem um efeito preocupante e perigoso sobre a seguridade social. Nesta perspectiva, o objetivo deste estudo é analisar os indicadores dos gastos público e privado no Brasil e países selecionados, no período de 2000 a 2014.

\section{Material e métodos}

Trata-se de uma pesquisa descritiva sobre gastos públicos e privados em saúde no Brasil e países selecionados (França, Itália, Espanha, Reino Unido e Portugal) por constituírem sistemas de saúde universais e passíveis de comparação através das seguintes dimensões: reformas no setor saúde, organização, financiamento das ações e serviços de saúde, prestação e regulação dos serviços.

Neste estudo, considerou-se para fins de análise a definição de 'gastos em saúde', ou 'despesas com saúde', como todos os gastos efetuados com a finalidade de recuperar, promover e manter a saúde dos indivíduos de certa população durante um período de tempo definido ${ }^{17}$.

A seleção dos dados secundários contemplou o período compreendido entre os anos 2000 e 2014, onde se encontram disponíveis as informações nas bases de dados consultadas. Para tanto, foram acessadas bases de domínio público, de diferentes fontes, quais sejam: Organização Mundial da Saúde (OMS), Ministério da Saúde (MS), Instituto Brasileiro de Geografia e Estatística (IBGE), além de revisão documental das publicações do Instituto de Pesquisa Econômica Aplicada (Ipea) obtidas via internet.

Para a análise dos gastos em saúde, foram extraídos e compilados dados da base da OMS (Global Health Expenditure Database) sobre gastos em saúde (público e privado) no Brasil, França, Itália, Espanha, Reino Unido e Portugal, referentes aos seguintes indicadores: despesa total per capita; despesa pública em saúde, em porcentagem do PIB; despesa pública em saúde, em porcentagem da despesa total em saúde; despesa pública em saúde, em proporção da despesa pública; despesa privada em porcentagem do PIB, desembolso direto (out-of-pocket) em porcentagem da despesa com saúde; e desembolso direto em porcentagem da despesa privada com saúde (quadro 1).

Quadro 1. Sistematização de indicadores para gastos públicos e privados com saúde

\begin{tabular}{lll}
\hline Indicador & Definição & Fonte \\
\hline Gasto total per capita & Gasto público e privado com consumo final de bens e & http://www.who.int/whosis/whosta- \\
& serviços de saúde, por habitante, no ano considerado. & t2006HealthFinancing.pdf \\
Gasto total em saúde & O total de despesas de saúde é a soma das despesas & http://www.who.int/whosis/whosta- \\
em \% do PIB & públicas e privadas de saúde. Abrange a prestação de & t2006HealthFinancing.pdf \\
& serviços de saúde (preventiva e curativa), atividades & \\
& de planejamento familiar, atividades de nutrição e & \\
& ajuda de emergência designada para a saúde, mas & \\
& não inclui provisão de água e saneamento. & \\
\hline
\end{tabular}




\begin{tabular}{|c|c|c|}
\hline $\begin{array}{l}\text { Gasto público em } \\
\text { saúde em \% do PIB }\end{array}$ & $\begin{array}{l}\text { Percentual do gasto público em saúde (consolidado } \\
\text { das três esferas do governo: federal, estadual e mu- } \\
\text { nicipal), em determinado espaço geográfico, no ano } \\
\text { considerado. Percentual do Produto Interno Bruto } \\
\text { (PIB) nacional que corresponde ao gasto público } \\
\text { federal com saúde, no ano considerado. }\end{array}$ & $\begin{array}{l}\text { http://tabnet.datasus.gov.br/cgi/ } \\
\text { idb2000/fqe06.htm }\end{array}$ \\
\hline $\begin{array}{l}\text { Gasto público em saú- } \\
\text { de em \% do gasto total } \\
\text { em saúde }\end{array}$ & $\begin{array}{l}\text { Percentual do gasto público que corresponde às } \\
\text { despesas com saúde, no ano considerado. }\end{array}$ & $\begin{array}{l}\text { https://gateway.euro.who.int/en/ } \\
\text { indicators }\end{array}$ \\
\hline $\begin{array}{l}\text { Gasto público em saú- } \\
\text { de em relação ao gasto } \\
\text { público total }\end{array}$ & $\begin{array}{l}\text { Este indicador é medido como parcela do gasto total } \\
\text { em saúde e em USD* per capita. } \\
\text { ^United States Dollar, que significa dólar dos Estados } \\
\text { Unidos. }\end{array}$ & $\begin{array}{l}\text { https://gateway.euro.who.int/en/ } \\
\text { indicators }\end{array}$ \\
\hline $\begin{array}{l}\text { Gasto privado em } \\
\text { saúde em \% do PIB }\end{array}$ & $\begin{array}{l}\text { Os gastos privados com saúde incluem gastos diretos } \\
\text { das famílias, seguros privados, doações de caridade } \\
\text { e pagamentos diretos de serviços por empresas } \\
\text { privadas. }\end{array}$ & $\begin{array}{l}\text { https://gateway.euro.who.int/en/ } \\
\text { indicators }\end{array}$ \\
\hline $\begin{array}{l}\text { Desembolso direto } \\
\text { (Out-of-pocket) em } \\
\% \text { do gasto total com } \\
\text { saúde }\end{array}$ & $\begin{array}{l}\text { Desembolsos são quaisquer gastos diretos das } \\
\text { famílias, incluindo gratificaçães e pagamentos em } \\
\text { espécie, para profissionais de saúde e fornecedores } \\
\text { de produtos farmacêuticos, aparelhos terapêuticos e } \\
\text { outros bens e serviços cuja intenção principal é con- } \\
\text { tribuir para a restauração ou melhoria do estado de } \\
\text { saúde. Faz parte do gasto privado em saúde. }\end{array}$ & $\begin{array}{l}\text { https://gateway.euro.who.int/en/ } \\
\text { indicators }\end{array}$ \\
\hline $\begin{array}{l}\text { Desembolso direto } \\
\text { (Out-of-pocket) em \% } \\
\text { do gasto privado em } \\
\text { saúde }\end{array}$ & $\begin{array}{l}\text { Refere-se ao pagamento direto ou desembolso direto } \\
\text { em proporção do gasto privado em saúde. }\end{array}$ & http://apps.who.int/nha/database \\
\hline
\end{tabular}

Fonte: Elaboração própria.

A moeda padrão utilizada foi o dólar devido à disponibilidade das informações por meio do banco de dados da Organização Mundial da Saúde e do Banco Mundial e, também, por possibilitar a comparação dos indicadores com outros países. Os dados foram tabulados e analisados pelo programa Microsoft ${ }^{\circledR}$ Office Excel $^{\circledR}$ 2010. Os resultados da pesquisa foram apresentados por meio de tabelas.

\section{Resultados e discussão}

Neste estudo foram analisados os dados referentes aos gastos em saúde - público e privado. A análise temporal demonstra heterogeneidade nos valores do indicador gasto público em saúde como porcentagem do gasto total em saúde quando comparados os países. Entretanto, observou-se uma certa estabilidade para cada país. O Brasil foi o país com menor participação do gasto público em saúde, $40 \%$ no primeiro ano (2000) e $46 \%$ no último ano analisado (2014). Em seguida, Portugal manteve a participação do gasto público entre $64 \%$ e $68 \%$. Os demais países ultrapassaram $70 \%$ do gasto público em saúde, com destaque para o Reino Unido, que alcançou $83 \%$ da participação do Estado nas ações e serviços públicos em saúde (tabela 1). 
Tabela 1. Gasto público em saúde como porcentagem do gasto total em saúde para países selecionados, 2000, 2008-2014

\begin{tabular}{lrrrrrrrr}
\hline Países & $\mathbf{2 0 0 0}$ & $\mathbf{2 0 0 8}$ & $\mathbf{2 0 0 9}$ & $\mathbf{2 0 1 0}$ & $\mathbf{2 0 1 1}$ & $\mathbf{2 0 1 2}$ & $\mathbf{2 0 1 3}$ & $\mathbf{2 0 1 4}$ \\
\hline Brasil & 40,3 & 43,8 & 44,4 & 45,8 & 45,2 & 44,3 & 45,1 & 46 \\
França & 79,4 & 77,3 & 77,5 & 77,5 & 77 & 77,2 & 77,1 & 78,2 \\
Itália & 72,1 & 76,9 & 77 & 77 & 75,1 & 75,3 & 75,6 & 75,6 \\
Portugal & 67,7 & 67,4 & 68,7 & 68,7 & 66,5 & 64 & 65,2 & 64,8 \\
Espanha & 71,6 & 74 & 75,7 & 75,1 & 73,9 & 71,7 & 71,5 & 70,9 \\
Reino Unido & 79,6 & 81,6 & 83,2 & 83,5 & 83,2 & 82,9 & 83,3 & 83,1 \\
\hline
\end{tabular}

Fonte: Elaboração própria, com base em dados da OMS.

Nota: Dados não disponíveis para o período de 2001 a 2007.

O gasto total per capita em saúde no Brasil alcançou US\$ 947. Os países que apresentaram os maiores valores foram França (US\$ 4.959) e Reino Unido (US\$ 3.934) (tabela 2). O gasto em saúde por habitante no Brasil foi menor do que os gastos apresentados pelos outros países, correspondendo aproximadamente a um gasto público diário de $\mathrm{R} \$ 2,60$ / dia per capita para cobrir as ações e serviços públicos de saúde da população brasileira, aquém do esperado em comparação com outros países que possuem sistemas universais de saúde. Ainda na tabela 2, é possível observar que a França teve o maior dispêndio em saúde, alcançando $11,5 \%$ do gasto total em saúde como proporção do PIB.

Tabela 2. Gasto total per capita e gasto total em saúde como porcentagem do PIB para países selecionados, 2014

\begin{tabular}{lrr}
\hline Países & Gasto total per capita $(\$$ US $)$ & Gasto total como porcentagem do PIB (\$US) \\
\hline Brasil & 947 & 8,3 \\
Espanha & 2.658 & 9 \\
Reino Unido & 3.935 & 9,1 \\
Itália & 3.258 & 9,2 \\
Portugal & 2.097 & 9,5 \\
França & 4.959 & 11,5 \\
\hline
\end{tabular}

Fonte: Elaboração própria, com base em dados da OMS.

Com relação ao gasto público em saúde como percentual do PIB, indicador que expressa a dimensão do gasto público com saúde no valor total da economia, em 2014, o Brasil destinou apenas 3,8\%. França e Reino
Unido foram os países que destinaram maior proporção do PIB com gasto público em saúde, 9\% e 7,6\%, respectivamente (tabela 3 ). Os demais países apresentaram um percentual acima ou pouco inferior a $9 \%$. 
Tabela 3. Gastos público e privado em saúde para países selecionados, 2014

\begin{tabular}{lrrrrrr}
\hline Indicadores & \multicolumn{7}{c}{ Países } \\
\cline { 2 - 7 } & Brasil & França & Itália & Portugal & Espanha & Reino Unido \\
\hline Gasto público em saúde como porcentagem do PIB & 3,8 & 9 & 7 & 6,2 & 6,4 & 7,6 \\
Gasto privado em saúde como porcentagem do PIB & 4,5 & 2,5 & 2,3 & 3,3 & 2,6 & 1,5 \\
$\begin{array}{l}\text { Gasto público em saúde como porcentagem do } \\
\text { gasto público total }\end{array}$ & 6,8 & 15,7 & 13,7 & 11,9 & 14,5 & 16,5 \\
$\begin{array}{l}\text { Gasto público em saúde como porcentagem do } \\
\text { gasto total em saúde }\end{array}$ & 46 & 78,2 & 75,6 & 64,8 & 70,9 & 83,1 \\
$\begin{array}{l}\text { Desembolso direto em porcentagem do gasto } \\
\text { total com saúde }\end{array}$ & 25,5 & 6,3 & 21,2 & 26,8 & 24 & 9,7 \\
$\begin{array}{l}\text { Desembolso direto em porcentagem do gasto } \\
\text { privado em saúde. }\end{array}$ & 47,2 & 29,1 & 86,9 & 76,3 & 82,4 & 57,7 \\
\hline
\end{tabular}

Fonte: Elaboração própria, com base em dados da OMS.

Segundo estimativas do IBGE, em 2012, o Brasil tinha um dispêndio total (público e privado) em saúde equivalente a $8,8 \%$ do $\mathrm{PIB}^{16}$. Esse percentual é bastante próximo ao de outros países da OCDE, como Reino Unido (8,4\%) e Espanha (8,5\%), que também possuem sistemas universais ${ }^{18}$. Contudo, o gasto público é muito baixo para que o País tenha, efetivamente, um sistema universal e atendimento integral.

Os demais países selecionados têm buscado ampliar o investimento de recursos públicos visando garantir o acesso dos seus cidadãos às ações e serviços de saúde. O percentual já ultrapassa 6\% do PIB há algum tempo, e, em muitos, supera 10\%. Estas comparações indicam que, apesar das discussões em torno da Emenda Constitucional no 29 , que prevê aumento dos recursos destinados ao SUS, o Brasil ainda tem que progredir na compreensão do custo e da efetiva necessidade de recursos para implantar um sistema, de fato, universal, integral e equitativo, de forma distinta às estabelecidas pela EC 95/16.

Quanto ao indicador gasto público em saúde como percentual do gasto total em saúde, em 2014, o Brasil investiu no subsistema público de saúde apenas $46 \%$ do total dos gastos em saúde. Países com sistemas universais despendem mais de $70 \%$ do gasto total com despesa pública em saúde. Por exemplo, Reino Unido e França, que foram os países que mais investiram no sistema público de saúde, gastaram, respectivamente, $83 \%$ e $78 \%$ (tabela 3 ).

No Brasil, no último ano analisado (2014), apenas $6,8 \%$ do gasto público foi destinado à saúde, pior desempenho quando comparado com os outros países. O Reino Unido e a França foram os países que mais investiram no sistema público de saúde, $16,5 \%$ e $15,7 \%$, respectivamente (tabela 3). Estes dados indicam que o gasto público com saúde do Brasil é baixo, em comparação com países com sistemas similares - isto é, universais e públicos -, que gastam, em média, o correspondente a $8 \%$ do PIB. O SUS destinou, em 2014 , apenas $3,9 \%$, o que resulta em um gasto per capita bastante baixo ${ }^{7,19}$.

O cenário explicitado ressalta o desafio do subfinanciamento público da saúde no Brasil, que impacta diretamente na dificuldade de abrangência das ações e serviços, e na qualidade da atenção à saúde. Embora, segundo Piola ${ }^{20}$, maiores níveis de gasto não impliquem necessariamente serviços de melhor qualidade, mais eficientes e equitativos, pois os modelos de financiamento e de 
organização dos sistemas de saúde exercem grande influência nos resultados em saúde.

Salienta-se que a recessão que assolou a Europa também impulsionou reformas estruturais e afetou a prioridade dada a determinadas políticas, especialmente referentes aos gastos públicos com saúde. Os EstadosMembros adotaram políticas de austeridade e reduziram substancialmente as despesas públicas. Os gastos com saúde per capita caíram, em termos reais, em 2010, em quase todos os países europeus, nomeadamente, a partir de uma taxa de crescimento médio anual de $4,6 \%$ ao ano, entre 2000 e 2009 , em direção a uma queda em gastos com saúde per capita de $0,6 \%$, em 2010. Contudo, mesmo diante deste cenário, os Estados-Membros da União Europeia (UE) dedicaram 9\% do seu PIB a gastos com saúde, significativamente $7,3 \%$, em 2000 , mas ligeiramente abaixo do pico de 9,2\%, em $2009^{21}$. Como resultado das medidas de austeridade, observa-se a queda nos gastos com saúde, a exemplo da porcentagem do PIB dedicada à saúde, estabilizada ou diminuída ligeiramente em muitos Estados-Membros da UE.

Entre os países analisados, o Brasil foi o que obteve maior proporção do gasto privado como proporção do PIB. No período, os valores mantiveram-se estáveis. A média foi de $4,5 \%$ da despesa privada como proporção do PIB. Seguindo a mesma tendência, Portugal apresentou elevada proporção do gasto privado. Reino Unido foi o país com melhor desempenho (1,6\%) (tabela 3). Quando comparado com o indicador gasto público como proporção do PIB, observa-se que, no Brasil, o gasto privado superou o público. Em todos os anos analisados, o Brasil apresentou o percentual do gasto privado superior ao público. Fato evidenciado, também, em outro estudo no qual os autores demonstraram que, no período correspondente entre 1975 e 2007, inverteram-se os percentuais do gasto nacional em saúde, assumindo o setor privado participação crescente em relação ao público ${ }^{22}$.

Uma análise sobre o financiamento da saúde no Brasil, nos anos que se seguiram à estabilidade econômica (1995-2012), organizados em cinco períodos (1995-1998, 19992002, 2003-2006, 2007-2010 e 2011-2012), evidenciou crescimento médio anual em todos os períodos. Indicou, do mesmo modo, um aumento de 79,8\% do quinto período em relação ao primeiro, correspondente a uma ampliação per capita de $R$ \$222,54 para $R$ \$ 400,06. O PIB apresentou um crescimento real em todos os períodos do estudo, acumulando um aumento médio anual de $61,4 \%$ do quinto em relação ao primeiro período. Os dados revelaram que os gastos com Ações e Serviços Públicos de Saúde (ASPS) cresceram mais do que o PIB, mas com uma participação percentual, com relação a este, quase linear, com média de 1,7\% em todo o período ${ }^{23}$.

Quanto ao financiamento privado nos países analisados, este corresponde a desembolsos diretos (out-of-pocket) ou a pré-pagamentos de planos e seguros privados de saúde. Nos países selecionados, observa-se uma mistura nos formatos de financiamento que merece atenção ${ }^{\mathbf{2 4}}$. Ao analisar o impacto do gasto em saúde no bem-estar das famílias e no acesso aos serviços de saúde, é particularmente relevante o indicador desembolso direto como proporção do gasto com saúde (público e privado). As análises empreendidas por este estudo demonstraram que, no Brasil, em 2014, esse indicador alcançou 25,5\% (tabela 3). França e Reino Unido foram os países com menor gasto das famílias em relação ao gasto total com saúde, respectivamente, $6,3 \%$ e 9,7\%, em 2014 . Roa ${ }^{25}$ identificou que, em 2013, o out-of-pocket era muito elevado e representava mais de $1 / 5$ dos gastos com saúde em cinco dos sete países incluídos na análise: Venezuela (66\%), Peru (35\%), Chile (32\%), Brasil (30\%) e Argentina (21\%).

No que se refere à participação do gasto direto das famílias no total do gasto privado em saúde nos países, verificou-se uma variação significativa em 2014: França com 29,1\%, menor valor apresentado; Brasil com 47,2\%; Itália apresentou 87\%; na sequência, Espanha $(82,4 \%)$ e Portugal (76,3\%) (tabela 3). Variação 
semelhante foi encontrada em outro estudo utilizando dados de 201124: Itália (92,7\%) e Espanha $(76,6 \%)$ foram os países que apresentaram os maiores gastos das famílias com saúde. Uma das formas mais comuns de gasto direto das famílias é o pagamento direto de medicamentos e serviços de saúde, exames e consultas, por exemplo. E quanto mais pobre é o país, maior é o gasto das famílias com saúde ${ }^{24,26}$. Em famílias com pessoas idosas e crianças, o gasto direto é mais elevado, possuindo um alto componente de medicamentos e serviços hospitalares ${ }^{25}$.

$\mathrm{O}$ pagamento direto restringe o acesso à saúde da população mais pobre, tendo em vista que as pessoas com menor poder aquisitivo possuem menor capacidade financeira para consumir os serviços de saúde 22,26. Alguns dados descritos em um dos relatórios técnicos do Ipea ${ }^{27}$ explicitaram que as famílias dos estratos inferiores de renda gastam, essencialmente, com medicamentos, e, à medida que cresce a renda per capita, os gastos das famílias tornam-se maiores com planos de saúde.

Em síntese, no conjunto dos indicadores analisados (gastos público e privado), o Brasil gasta menos do que os países selecionados para comparação, ocorrendo o mesmo em relação a países da América Latina que não possuem sistemas universais de saúde ${ }^{24,25}$. Os resultados deste estudo põem em evidência o desafio de construir uma dupla agenda: fortalecer o SUS, melhorando o financiamento, mas, também, regular o mercado, em particular, dos planos de saúde, tendo como eixo norteador a universalidade, de um lado, e a redução dos gastos das famílias, dos trabalhadores e dos empregadores com bens e serviços privados de saúde, do outro ${ }^{\mathbf{2 8}}$.

\section{Considerações finais}

Este artigo buscou contemplar a identificação e breve análise de alguns indicadores referentes aos gastos públicos e privados no Brasil. Os principais resultados apontam que os gastos públicos em saúde decresceram ou não apresentaram aumento no período estudado, e que os gastos privados em saúde se mantiveram estáveis. Outra questão que merece destaque: o excesso de incentivos governamentais para o mercado privado de saúde contribui para a redução do gasto público, mantendo o subfinanciamento do sistema público de saúde brasileiro, além de dificultar a implementação do SUS como um sistema de saúde universal, conforme previsto na Constituição de 198816,29.

Se não bastasse o subfinanciamento do sistema, desde a sua criação pela Constituição de 1988, ainda há a concorrência com o setor privado, que vê no SUS a possibilidade de ampliação do capital financeiro. Atrelado a isto, conforme já mencionado, o setor privado é beneficiado pelo Estado, por meio de incentivos financeiros, mais evidentes a partir de $2014^{\mathbf{1 6}}$. Percebe-se que a situação de subfinanciamento do SUS pode ser considerada satisfatória para os que valorizam apenas a questão do equilíbrio fiscal, dado que são anos em que o expressivo volume de recursos aplicados na saúde foi menor do que o necessário. A superação do subfinanciamento é improvável na ausência de maior envolvimento dos usuários e de outros setores organizados ${ }^{30}$. Ademais, os dados demonstram que o gasto com a saúde privada é relevante no orçamento familiar. Tais achados apontam para uma redução do papel do Estado como provedor e financiador das ações e serviços públicos de saúde. Sobre isto, além das fontes e da estrutura do financiamento, o nível de gastos com saúde condiciona e determina em que medida o sistema de saúde responde às necessidades da população ${ }^{25}$.

Em síntese, a estruturação de um sistema público universal depende das expectativas e possibilidades da repartição público-privada no financiamento do sistema e oferta de serviços de saúde, bem como da capacidade e interesse dos agentes do Estado para regular essa relação, visando à priorização do sistema público. Enquanto persistir o dilema entre a universalidade do SUS diante 
da sua dificuldade de financiamento público, recrudesce a necessidade de envidar esforços para assegurar a efetiva priorização do SUS no gasto público e o cumprimento dos seus princípios basilares.

\section{Colaboradores}

Figueiredo JO contribuiu para elaboração do artigo com as seguintes atividades:

\section{Referências}

1. Brasil. Constituição da República Federativa do Brasil de 1988. Brasília, DF: Senado Federal; 1988.

2. Paim JS. O que é SUS. Rio de Janeiro: Fiocruz; 2009.

3. Brasil. Lei no 8.080, de 19 de setembro de 1990. Dispõe sobre as condições para a promoção, proteção e recuperação da saúde, a organização e o funcionamento dos serviços correspondentes e dá outras providências. Diário Oficial da União. 19 Set 1990.

4. Brasil. Lei $\mathrm{n}^{\circ}$ 8.142, de 28 de dezembro de 1990. Dispõe sobre a participação da comunidade na gestão do Sistema Único de Saúde (SUS) e sobre as transferências intergovernamentais de recursos financeiros na área da saúde e dá outras providências. Diário Oficial da União. 28 Dez 1990.

5. Brasil. Lei Complementar $n^{\circ} 141$, de 13 de janeiro de 2012. Diário Oficial da União. 13 Jan 2012.

6. Vieira FS, Benevides RPS. O direito à saúde no Brasil em tempos de crise econômica, ajuste fiscal e refor- concepção, delineamento, coleta, análise e processamento dos dados, redação da versão final do artigo. Prado NMBL contribuiu para elaboração do artigo com as seguintes atividades: coleta, análise e processamento dos dados, redação da versão final do artigo. Medina MG e Paim JS contribuíram para elaboração do artigo com as seguintes atividades: contribuição significativa na revisão crítica do conteúdo e participação da aprovação da versão final do manuscrito.

ma implícita do Estado. Rev. Est. Pesq. Amer. 2016; 10(3):1-28.

7. Marques RM, Piola SF, Ocké-Reis CO. Desafios e perspectivas futuras no financiamento do SUS. In: Marques RM, Piola SF, Roa AC, organizadores. Sistema de Saúde no Brasil: organização e financiamento. Rio de Janeiro: Abres; Brasília, DF: MS; 2016.

8. Análise Política em Saúde. Crise no Brasil: quais os impactos na saúde da população. Bol. Projeto Análise de Políticas de Saúde no Brasil [internet]. 2016 nov-dez [acesso em 2018 maio 20]; 2(8). Disponível em: http://www.analisepoliticaemsaude.org/oaps/ boletim/edicao/8.

9. Maresso A, Mladovsky P, Thomson S et al. Economics crisis, health systems and health in Europe. Copenhagen: WHO; 2015.

10. Vieira FS. Crise Econômica, austeridade fiscal e saúde: que lições podem ser aprendidas? Brasília, DF: Ipea; 2016. 
11. OPSS. Observatório Português dos Sistemas de Saúde. Saúde: procuram-se novos caminhos. Relatório de primavera 2016. Portugal: OPSS; 2016.

12. Conill EM. A importância da continuidade dos sistemas nacionais europeus para as políticas de saúde na América latina. Cad Saúde Pública. 2014; 30(11):22532255.

13. Karanikolos M, Heino P, McKee M, et al. Effects of the global financial crisis on health in high-income OECD countries: a narrative review. Int. J. Health Serv. 2016; 46(2):208-240.

14. Mucci N, Giorgi G, Roncaioli M, et al. The correlation between stress and economic crisis: a systematic review. Neuropsychiatric Dis. Treat. 2016; 12:983-993.

15. Borges FT, Moimaz SAS, Siqueira CE, et al. Anatomia da privatização neoliberal do Sistema de Saúde: O papel das Organizações Sociais de Saúde. São Paulo: Cultura Acadêmica; 2012.

16. Melo MFGC. Relações público-privadas no sistema de saúde brasileiro [tese]. Campinas: Unicamp; 2017 [acesso em 2018 abr 20]. 206 p. Disponível em: http://repositorio.unicamp.br/bitstream/REPOSIP/330343/1/ Melo_MariaFernandaGodoyCardosoDe_D.pdf.

17. Coelho TCB, Scatena JHG. Financiamento do SUS. In: Paim JS, Almeida-Filho N, organizadores. Saúde Coletiva: Teoria e Prática. Rio de Janeiro: MedBook; 2014. p. 271-286.

18. Instituto Brasileiro de Geografia e Estatística. Microdados sobre saúde. Rio de Janeiro: IBGE; 2012 [acesso em 2018 abr 10]. Disponível em: https://www.ibge. gov.br/estatisticas-novoportal/sociais/saude.html.

19. World Health Organization. Global Health Observatory Data Repository [internet]. Geneva: WHO; 2016 [acesso em 2018 mar 10]. Disponível em: http://apps. who.int/gho/data/node.country.country.

20. Piola S. Financiamento da saúde em países selecionados da América Latina. In: Rodrigues VA, organizador. Observatório internacional de capacidades hu- manas, desenvolvimento e políticas públicas: estudos e análises. 1. ed. Brasília, DF: UNB; 2013. p. 193-211.

21. Quaglio G, Karapiperis T, Woensel LV, et al. Austerity and health in Europe. Health Policy [internet]. 2013 nov [acesso em 2018 maio 10]; 113(1-2):13-19. Disponível em: https://www.sciencedirect.com/science/ article/pii/S0168851013002303.

22. Silva CL, Rotta CV. O dilema da universalidade e financiamento público do Sistema Único de Saúde no Brasil. Textos Contextos [internet]. 2012 ago-dez [acesso em 2018 maio 10]; 11(2):333-345. Disponível em: http://revistaseletronicas.pucrs.br/ojs/index. php/fass/article/viewFile/12126/8642.

23. Soares A, Santos NR. Financiamento do Sistema Único de Saúde nos governos FHC, Lula e Dilma. Saúde debate [internet]. 2014 jan-mar [acesso em 2018 maio 10]; 38(100):18-25. Disponível em: http://www.scielo.br/scielo.php?pid=S0103$-11042014000100018 \&$ script=sci_abstract $\&$ tlng=pt.

24. Marques RM. O direito à saúde no mundo. In. Marques RM, Piola SF, Roa AC, organizadores. Sistema de saúde no Brasil: organização e financiamento. Rio de Janeiro: ABrES; Brasília, DF: MS; 2016. p. 11-36.

25. Roa AC. Financiamento dos sistemas de saúde na América do Sul. In. Marques RM, Piola SF, Roa AC, organizadores. Sistema de saúde no Brasil: organização e financiamento. Rio de Janeiro: ABrES; Brasília, DF: MS; 2016. p. 75-98.

26. Organização Mundial da Saúde. Relatório Mundial da Saúde: o caminho para a cobertura universal. Genebra: OMS; 2010 [acesso em 2018 maio 20]. Disponível em: http://www.who.int/whr/2010/whr10_pt.pdf.

27. Diniz CPB, Servo SML, Piola FS, et al. Gasto das famílias com saúde no Brasil: evolução e debate sobre gasto catastrófico [internet]. Brasília, DF: Ipea; 2007 [acesso em 2018 maio 20]. Disponível em: http:// ipea.gov.br/agencia/images/stories/PDFs/livros/10_ Cap04.pdf.

28. Marques RM, Piola SF. O financiamento da saúde 
depois de 25 anos de SUS. In: Rizotto MLF, Costa A, organizadoras. 25 anos de direito universal à saúde. Rio de Janeiro: Cebes; 2014. p. 178-195.

29. Sestelo JAF. Planos e seguros de saúde do Brasil de 2000 a 2015 e a dominância financeira [tese]. Rio de Janeiro: UFRJ; 2017.
30. Gomes FBC. Impasses no financiamento da saúde no Brasil: da constituinte à regulamentação da Emenda 29/00. Saúde debate. 2014 jan-mar; 38(100):6-17.

Recebido em 11/07/2018

Aprovado em 23/09/2018

Conflito de interesses: inexistente

Suporte financeiro: não houve 